\title{
Dynamics of competing systems in general heterogeneous environments
}

\section{Benlong $\mathrm{Xu}^{*}$ and Zhenzhang $\mathrm{Ni}$}

\section{*Correspondence:} bxu@shnu.edu.cn

Department of Mathematics, Shanghai Normal University, Shanghai, 200234, P.R. China (c) $2015 \mathrm{Xu}$ and $\mathrm{Ni}$. This article is distributed under the terms of the Creative Commons Attribution 4.0 International License (http://creativecommons.org/licenses/by/4.0/), which permits unrestricted use, distribution, and reproduction in any medium, provided you give appropriate credit to the original author(s) and the source, provide a link to the Creative Commons license, and indicate if changes were made.

\begin{abstract}
In this paper, we address the question of the dynamics of the systems for two competing species in general heterogeneous environment with lethal boundary conditions. The existence and uniqueness of the positive steady-state solution are established under suitable conditions. Finally, we obtain global asymptotic stability of the positive steady-state solution for weak competition situation.
\end{abstract}

Abstract

MSC: $35 \mathrm{~K} 57 ; 92 \mathrm{D} 25$

Keywords: global asymptotic stability; competing systems; positive steady-state solution; coexistence

\section{Introduction}

The model we consider has the general form

$$
\begin{cases}\frac{\partial u}{\partial t}=\mu_{1} \Delta u+u[a(x)-b(x) u-c(x) v] & \text { in } \Omega \times[0, \infty), \\ \frac{\partial v}{\partial t}=\mu_{2} \Delta v+v[d(x)-e(x) u-f(x) v] & \text { in } \Omega \times[0, \infty), \\ u=v=0 & \text { on } \partial \Omega \times[0, \infty), \\ u(x, 0)=u^{0}(x), \quad v(x, 0)=v^{0}(x) & \text { in } \partial \Omega,\end{cases}
$$

where $a(x), b(x), d(x), f(x)>0, c(x), e(x) \geq 0$, all belong to the Hölder space $C^{\alpha}(\bar{\Omega})$ for some constant $0<\alpha<1$, and $\Omega \subseteq \mathbb{R}^{N}$ is a bounded domain with $C^{2+\alpha}$ a smooth boundary. The variables $u, v$ represent population densities of the competing species. The boundary condition describes the situation that the boundary of $\Omega$ is lethal to the species.

When the coefficients $a(x), b(x), c(x), d(x), e(x), f(x)$ are constants (homogeneous environment), the system (1.1) has been studied extensively in past years; see, for example, [1-6] and the references therein. But in the real world, the environments are usually heterogenous, and so it is more reasonable to assume that the coefficients in the system (1.1) are more general functions [7]. Recently, $\mathrm{He}$ and $\mathrm{Ni}$ studied the dynamics of a competition model in some heterogeneous environments with Neumann boundary conditions $[8,9]$. Álvarez-Caudevilla et al. considered a cooperative reaction-diffusion system in a spatiotemporally degenerate environment [10].

To study the dynamics of the system (1.1), we will consider its steady-state equation,

$$
\begin{cases}\mu_{1} \Delta u+u[a(x)-b(x) u-c(x) v]=0 & \text { in } \Omega \times[0, \infty), \\ \mu_{2} \Delta v+v[d(x)-e(x) u-f(x) v]=0 & \text { in } \Omega \times[0, \infty), \\ u=v=0 & \text { on } \partial \Omega \times[0, \infty)\end{cases}
$$


Let $\lambda_{1}>0$ denote the principal eigenvalue for the problem

$$
\begin{cases}\Delta \phi+\lambda \phi=0 & \text { in } \Omega, \\ \phi=0 & \text { on } \partial \Omega .\end{cases}
$$

For any $A(x) \in C^{\alpha}(\bar{\Omega})$, it is well known that

$$
\begin{cases}\mu \Delta \theta+\theta(A(x)-\theta)=0 & \text { in } \Omega, \\ \theta=0 & \text { on } \partial \Omega,\end{cases}
$$

has a unique positive solution $\theta \in C^{2+\alpha}(\bar{\Omega})$ if $A(x)>\mu \lambda_{1}$ for all $x \in \bar{\Omega}$ [5]. We denote this unique positive solution by $\theta_{\mu, A(x)}$.

In the rest of the paper, we always assume that

$$
a(x)>\mu_{1} \lambda_{1}, \quad d(x)>\mu_{2} \lambda_{1} \quad \text { for all } x \in \bar{\Omega},
$$

and denote

$$
\bar{u}(x)=\frac{\theta_{\mu_{1}, a(x)}}{\min _{x \in \bar{\Omega}} b(x)}, \quad \bar{v}(x)=\frac{\theta_{\mu_{2}, d(x)}}{\min _{x \in \bar{\Omega}} f(x)}, \quad x \in \bar{\Omega} .
$$

Now we state the existence result for steady-state solutions.

Theorem 1.1 (Existence) If

$$
a(x)>\mu_{1} \lambda_{1}+c(x) \bar{v}(x), \quad d(x)>\mu_{2} \lambda_{1}+e(x) \bar{u}(x),
$$

for all $x \in \bar{\Omega}$, then the system (1.2) has a positive steady-state solution $(\tilde{u}(x), \tilde{v}(x))$ with $\tilde{u}(x), \tilde{v}(x) \in C^{2+\alpha}(\bar{\Omega})$.

Now we suppose further that the two competitors in (1.1) are under weak competition in the sense that $c(x), e(x)>0$ are small such that

$$
\frac{c(x)}{\min _{x \in \bar{\Omega}} f(x)}<\frac{a(x)}{d(x)}, \quad \frac{e(x)}{\min _{x \in \bar{\Omega}} b(x)}<\frac{d(x)}{a(x)} \quad \text { for all } x \in \bar{\Omega} .
$$

We denote

$$
\begin{aligned}
& \bar{\zeta}=\theta_{\mu_{1}, a(x)}, \quad \bar{\eta}=\theta_{\mu_{2}, d(x)}, \\
& \left.\underline{\zeta}=\theta_{\mu_{1},(a(x)-c(x)} \frac{d(x)}{\min _{x \in \bar{\Omega}} f(x)}\right), \quad \underline{\eta}=\theta_{\mu_{2},\left(d(x)-e(x) \frac{a(x)}{\min _{x \in \bar{\Omega}} b(x)}\right)},
\end{aligned}
$$

which are all positive functions in $\Omega$.

The following theorem gives a sufficient conditions for uniqueness of coexistence solution in suitable weak competition situations.

Theorem 1.2 (Uniqueness) Assume that all the hypotheses of Theorems 1.1 and (1.8) are satisfied. If 


$$
\frac{c^{2}(x) \bar{\zeta}}{b^{2}(x) \underline{\eta}}+2 \frac{c(x) e(x)}{b(x) f(x)}+\frac{e^{2}(x) \bar{\eta}}{f^{2}(x) \underline{\zeta}}<4, \quad x \in \bar{\Omega},
$$

then the steady-state solution $(\tilde{u}(x), \tilde{v}(x))$ of $(1.2)$ is unique.

Remark 1.1 For fixed functions $a(x)>0, d(x)>0$, hypothesis (1.10) will be satisfied for $c(x), e(x) \geq 0$ sufficiently small. This is true because $\zeta$ (resp. $\eta$ ) increases as $c(x)$ (resp. $e(x)$ ) decreases for $x \in \Omega$. Thus $\frac{e^{2}(x) \bar{\eta}}{f^{2}(x) \underline{\underline{\zeta}}}$ (resp. $\frac{c^{2}(x) \bar{\zeta}}{b^{2}(x) \underline{\underline{\eta}}}$ ) decreases as $c(x)$ (resp. $\left.e(x)\right)$ decreases.

Finally, we state our dynamics results for the system (1.1).

Theorem 1.3 (Global asymptotic stability) Assume that the hypotheses of Theorem 1.2 are satisfied. Let $(u(x, t), v(x, t))$ be a solution of the initial boundary value problem (1.1) with both $u^{0}, v^{0} \geq 0, \equiv 0$ in $C^{\alpha}(\bar{\Omega}), 0<\alpha<1$, and vanishing on $\partial \Omega$, then

$$
(u(x, t), v(x, t)) \rightarrow(\tilde{u}(x), \tilde{u}(x)) \quad \text { as } t \rightarrow \infty
$$

uniformly in $\bar{\Omega}$.

This paper is organized as follows: Theorem 1.1 and Theorem 1.2 are proved in Section 2. Theorem 1.3 is established in Section 3 by proving a more general theorem.

\section{Proof of Theorem 1.1 and Theorem 1.2}

Proof of Theorem 1.1 Let $\phi(x)$ be a positive eigenfunction of the principal eigenvalue $\lambda_{1}$ for the eigenvalue problem (1.3). Choose $r_{1}>0$ sufficiently small,

$$
\begin{array}{r}
\mu_{1} \Delta\left(r_{1} \phi(x)\right)+r_{1} \phi(x)\left[\left(a(x)-b(x) r_{1} \phi(x)-c(x) v\right]\right. \\
\quad \geq r_{1} \phi(x)\left[\left(a(x)-\mu_{1} \lambda_{1}-b(x) r_{1} \phi(x)-c(x) \bar{v}\right] \geq 0\right.
\end{array}
$$

and

$$
\begin{aligned}
\mu_{1} \Delta \bar{u}+\bar{u}[a(x)-b(x) \bar{u}-c(x) v] \\
\quad=\bar{u}\left[\left(1-\frac{b(x)}{\min _{x \in \bar{\Omega}} b(x)}\right) \theta_{\mu_{1}, a(x)}-c(x) v\right] \leq 0
\end{aligned}
$$

for all $0 \leq v \leq \bar{v}$. So, $\left(\bar{u}, r_{1} \phi(x)\right)$ is a set of upper and lower solutions for $u$ in (1.2).

Similarly, choose $r_{2}>0$ sufficiently small, $\left(\bar{v}, r_{2} \phi(x)\right)$ is a set of upper and lower solutions for $v$ in (1.1). By the coupled upper and lower theorem [5], the system (1.2) has a steadystate solution $(\tilde{u}(x), \tilde{v}(x))$ with $\tilde{u}(x), \tilde{v}(x)>0$ for $x \in \Omega$.

Proof of Theorem 1.2 Assume that $\left(\tilde{u}_{1}(x), \tilde{v}_{1}(x)\right),\left(\tilde{u}_{2}(x), \tilde{v}_{2}(x)\right)$ are two strictly positive steady-state solutions of the system (1.1) in $\Omega$.

Let

$$
\begin{aligned}
& p(x)=\tilde{u}_{1}(x)-\tilde{u}_{2}(x), \quad q(x)=\tilde{v}_{1}(x)-\tilde{v}_{2}(x), \\
& I_{1}=b(x) \tilde{u}_{2}(x) p(x)+c(x) \tilde{u}_{2}(x) q(x)
\end{aligned}
$$




$$
I_{2}=e(x) \tilde{v}_{1}(x) p(x)+f(x) \tilde{v}_{1}(x) q(x)
$$

then

$$
\begin{cases}\mu_{1} \Delta p(x)+\left[a(x)-b(x) \tilde{u}_{1}(x)-c(x) \tilde{v}_{1}(x)\right] p(x)-I_{1}=0 & \text { in } \Omega, \\ \mu_{2} \Delta q(x)+\left[d(x)-e(x) \tilde{u}_{2}(x)-f(x) \tilde{v}_{2}(x)\right] q(x)-I_{2}=0 & \text { in } \Omega, \\ p(x)=q(x)=0 & \text { on } \partial \Omega .\end{cases}
$$

Since $\tilde{u}_{1}(x)$ is a strictly positive solution of

$$
\begin{cases}\mu_{1} \Delta \psi+\left[a(x)-b(x) \tilde{u}_{1}(x)-c(x) \tilde{v}_{1}(x)\right] \psi+\alpha \psi=0 & \text { in } \Omega \\ \psi=0 & \text { on } \partial \Omega\end{cases}
$$

with $\alpha=0$, the number $\alpha=0$ must be the smallest eigenvalue of the above problem. Moreover, by the variational properties, we have

$$
\int_{\Omega} z\left(-\mu_{1} \Delta z-\left[a(x)-b(x) \tilde{u}_{1}(x)-c(x) \tilde{u}_{2}(x)\right] z\right) d x \geq 0
$$

for any $z \in C^{2}(\bar{\Omega})$ which vanishes on $\partial \Omega$. Similarly, since $\tilde{v}_{2}(x)$ is strictly positive solution of

$$
\begin{cases}\mu_{2} \Delta \psi+\left[d(x)-e(x) \tilde{u}_{2}(x)-f(x) \tilde{v}_{2}(x)\right] \psi+\alpha \psi=0 & \text { in } \Omega \\ \psi=0 & \text { on } \partial \Omega\end{cases}
$$

with $\alpha=0$, the number $\alpha=0$ must be the smallest eigenvalue of the above problem. Moreover,

$$
\int_{\Omega} z\left(-\mu_{2} \Delta z-\left[d(x)-e(x) \tilde{u}_{2}(x)-f(x) \tilde{v}_{2}(x)\right] z\right) d x \geq 0
$$

for any $z \in C^{2}(\bar{\Omega})$ which vanishes on $\partial \Omega$. Multiplying the first equation of (2.1) by $-p(x)$, the second by $-q(x)$, integrating over $\Omega$, and adding, we deduce from (2.2) and (2.3) that

$$
\int_{\Omega}\left[b(x) \tilde{u}_{2}(x) p^{2}(x)+\left(c(x) \tilde{u}_{2}(x)+e(x) \tilde{v}_{1}(x)\right) p(x) q(x)+f(x) \tilde{v}_{2}(x) q^{2}(x)\right] d x \leq 0
$$

By a comparison of scalar equations using upper and lower solutions we readily obtain, for $x \in \Omega$,

$$
\begin{aligned}
\frac{\zeta}{b(x)} & \leq \tilde{u}_{1}, \tilde{u}_{2} \leq \frac{\bar{\zeta}}{b(x)} \\
\frac{\underline{\eta}}{f(x)} & \leq \tilde{v}_{1}, \tilde{v}_{2} \leq \frac{\bar{\eta}}{f(x)}
\end{aligned}
$$

From (2.5), we have

$$
\frac{f(x) c^{2}(x) \bar{\zeta}}{b(x) \underline{\eta}}+2 c(x) e(x)+\frac{b(x) e^{2}(x) \bar{\eta}}{f(x) \underline{\zeta}}>c^{2}(x) \frac{\tilde{u}_{2}(x)}{\tilde{v}_{1}(x)}+2 c(x) e(x)+e^{2}(x) \frac{\tilde{v}_{1}(x)}{\tilde{u}_{2}(x)}
$$


in $\Omega$. It follows from (1.10) that

$$
c^{2}(x) \frac{\tilde{u}_{2}(x)}{\tilde{v}_{1}(x)}+2 c(x) e(x)+e^{2}(x) \frac{\tilde{v}_{1}(x)}{\tilde{u}_{2}(x)}<4 b(x) f(x) \quad \text { in } \Omega .
$$

Then it is easy to see that the quadratic expression in the integrand of (2.4) is positive definite for each $x \in \Omega$. Consequently, we must have $p(x)$ and $q(x)$ identically equal to zero in $\Omega$. That is, $\left(\tilde{u}_{1}(x), \tilde{v}_{2}(x)\right) \equiv\left(\tilde{u}_{2}(x), \tilde{v}_{2}(x)\right)$ in $\Omega$.

\section{Proof of Theorem 1.3}

Now we are in a position to prove Theorem 1.3. Note that, by Theorem 1.2 and the assumptions of Theorem 1.3, problem (1.2) has a unique positive solution $(\tilde{u}(x), \tilde{u}(x))$. Then we will establish Theorem 1.3 by proving the following theorem without the assumption (1.10).

Theorem 3.1 Assume the hypotheses of Theorem 1.1 and that problem (1.2) has a unique positive solution $(\tilde{u}(x), \tilde{u}(x))$ in $\Omega$, then $(\tilde{u}(x), \tilde{u}(x))$ is globally asymptotically stable in the following sense. Let $(u(x, t), v(x, t))$ be a solution of the initial boundary value problem (1.1) with both $u^{0}, v^{0} \geq 0, \equiv 0$ in $C^{\alpha}(\bar{\Omega}), 0<\alpha<1$, and vanishing on $\partial \Omega$, then

$$
(u(x, t), v(x, t)) \rightarrow(\tilde{u}(x), \tilde{u}(x)) \quad \text { as } t \rightarrow \infty,
$$

uniformly in $\bar{\Omega}$.

Proof For convenience, we introduce the following notation: If $w \in C^{1}(\bar{\Omega}), w(x)>0$ for all $x \in \Omega$, and $\partial w / \partial v<0$ everywhere on $\partial \Omega$, we write $w \gg 0$. If $w, z \in C^{1}(\bar{\Omega})$, we write $w \ll z$ if $z-w \gg 0$. We first prove the theorem under the additional conditions $u^{0}, v^{0} \in C^{1}(\bar{\Omega})$,

$$
u^{0} \gg 0, \quad v^{0} \gg 0,
$$

and for all $x \in \bar{\Omega}$,

$$
u^{0} \leq \bar{u}, \quad v^{0} \leq \bar{v}
$$

where $\bar{u}$ and $\bar{v}$ are defined in (1.6).

Let $\phi_{1}$ be the positive eigenfunction of the principal eigenvalue in (1.3). Choose $\epsilon>0$ small such that

$$
\epsilon \phi_{1}(x) \leq u^{0}(x), \quad \epsilon \phi_{1}(x) \leq v^{0}(x)
$$

and

$$
\begin{aligned}
& a(x)>\mu_{1} \lambda_{1}+c(x) \bar{v}+b(x) \epsilon \phi_{1}(x), \\
& d(x)>\mu_{2} \lambda_{1}+e(x) \bar{u}+f(x) \epsilon \phi_{1}(x),
\end{aligned}
$$

for all $x \in \bar{\Omega}$. If we let $\underline{u}=\underline{v}=\epsilon \phi_{1}$, then

$$
\mu_{1} \Delta \bar{u}+\bar{u}[a(x)-b \bar{u}-c(x) \underline{v}]=\bar{u}\left[\left(1-\frac{b(x)}{\min _{x \in \bar{\Omega}} b(x)}\right) \theta_{\mu_{1}, a(x)}-c(x) \underline{v}\right]<0,
$$


for all $x \in \Omega$; and from (3.4), we have

$$
\mu_{2} \Delta \underline{v}+\underline{v}[d(x)-e \bar{u}-f(x) \underline{v}]=\underline{v}\left[d(x)-\mu_{2} \lambda_{1}-e(x) \bar{u}-f(x) \underline{v}\right]>0,
$$

on $\Omega$. Similarly, we have

$$
\begin{aligned}
& \mu_{1} \Delta \underline{u}+\underline{u}[a(x)-b(x) \underline{u}-c(x) \bar{v}]>0, \\
& \mu_{2} \Delta \bar{v}+\bar{v}[d(x)-e(x) \underline{u}-f(x) \bar{v}]<0 .
\end{aligned}
$$

By Theorem 1.3 in [1] (also see Pao [11], Section 10.5), the conclusion of the theorem follows from the uniqueness assumption, the inequalities $\underline{u}(x) \leq u^{0} \leq \bar{u}(x), \underline{v}(x) \leq v^{0} \leq \bar{v}(x)$, $x \in \bar{\Omega}$, and a comparison with solutions of the differential system (1.1) with initial conditions replaced at the steady-state upper lower solutions $(\bar{u}(x), \underline{v}(x))$.

We next remove condition (3.2) on the initial functions $u^{0}(x), v^{0}(x)$. First, observe that there exists large $K>1$, such that

$$
u^{0}(x) \leq K \bar{u}, \quad V^{0}(x) \leq K \bar{\nu},
$$

on $\Omega$. Define $(\bar{U}(x, t), \underline{V}(x, t))$ to be the solution of problem (1.1) with initial conditions replaced with

$$
(\bar{U}(x, 0), \underline{V}(x, 0))=(K \bar{u}, 0) .
$$

It is clear that $\underline{V} \equiv 0, \bar{U}$ is non-negative in $\Omega \times[0, \infty)$ and

$$
\lim _{t \rightarrow \infty} \bar{U}(x, t)=\bar{U}^{\star}(x) \quad \text { for } x \in \Omega
$$

where $\bar{U}^{\star}(x)$ is the unique positive solution of the problem

$$
\mu_{1} \Delta z+z[a(x)-b(x) z]=0 \quad \text { in } \Omega, \quad z=0 \quad \text { on } \partial \Omega
$$

Moreover, the convergence above is monotone, because $\bar{U}(x, 0), \underline{V}(x, 0)$ satisfies

$$
\begin{aligned}
& \mu_{1} \Delta \bar{U}(x, 0)+\bar{U}(x, 0)[a(x)-b(x) \bar{U}(x, 0)-c(x) \underline{V}(x, 0)] \\
& \quad=(\bar{U}(x, 0))^{2}\left[\frac{\min _{x \in \bar{\Omega}} b(x)}{K}-b\right]<0, \\
& \mu_{2} \Delta \underline{V}(x, 0)+\underline{V}(x, 0)[d(x)-e(x) \bar{U}(x, 0)-f(x) \underline{V}(x, 0)]=0 .
\end{aligned}
$$

The convergence in (3.5) is also in $C^{1}(\bar{\Omega})$ norm by using the $W^{2, p}$ estimates, compact embedding, and (1.1). Similarly, define $(\underline{U}(x, t), \bar{V}(x, t))$ to be the solution of problem (1.1) with initial conditions replaced with

$$
(\underline{U}(x, 0), \bar{V}(x, 0))=(0, K \bar{v}) \text {. }
$$


We have $\underline{U} \equiv 0, \bar{V}$ is non-negative in $\Omega \times[0, \infty)$, and we have monotone $C^{1}(\bar{\Omega})$ convergence,

$$
\lim _{t \rightarrow \infty} \bar{V}(x, t)=\bar{V}^{\star}(x)
$$

where $\bar{V}^{\star}(x)$ is the unique positive solution of the problem

$$
\mu_{2} \Delta z+z[d(x)-f(x) z] \quad \text { in } \Omega,\left.\quad z\right|_{\partial \Omega}=0
$$

On the other hand, one readily verifies that the functions $\underline{U}(x, t), \bar{U}(x, t), \underline{V}(x, t), \bar{V}(x, t)$ satisfy

$$
\begin{aligned}
& \mu_{1} \Delta \bar{U}+\bar{U}[a(x)-b(x) \bar{U}-c(x) \underline{V}]-\partial \bar{U} / \partial t<0, \\
& \mu_{2} \Delta \underline{V}+\underline{V}[d(x)-e(x) \bar{U}-f(x) \underline{V}]-\partial \underline{V} / \partial t \geq 0, \\
& \mu_{2} \Delta \bar{V}+\bar{V}[d(x)-e(x) \underline{U}-f(x) \bar{V}]-\partial \bar{V} / \partial t<0, \\
& \mu_{1} \Delta \underline{U}+\underline{U}[a(x)-b(x) \underline{U}-c(x) \bar{V}]-\partial \underline{U} / \partial t \geq 0,
\end{aligned}
$$

for $(x, t) \in \Omega \times(0, \infty)$, and

$$
\begin{aligned}
& 0=\underline{U}(x, 0) \leq u^{0}(x) \leq \bar{U}(x, 0)=K \bar{u}, \\
& 0=\underline{V}(x, 0) \leq \nu^{0}(x) \leq \bar{V}(x, 0)=K \bar{v},
\end{aligned}
$$

for $x \in \bar{\Omega}$. From the comparison theorems, we assert that

$$
\begin{aligned}
& 0=\underline{U}(x, t) \leq u^{0}(x, t) \leq \bar{U}(x, t), \\
& 0=\underline{V}(x, t) \leq v^{0}(x, t) \leq \bar{V}(x, t),
\end{aligned}
$$

for $(x, t) \in \Omega \times[0, \infty)$. We next observe that $\mu_{1} \Delta \bar{u}+\bar{u}[a(x)-b(x) \bar{u}]<0$ in $\Omega,\left.\bar{u}\right|_{\partial \Omega}=0$, thus $\bar{u}=\theta_{\mu_{1}, a(x)} / \min _{x \in \bar{\Omega}} b(x)$ is a strict upper solution of the problem (3.6). Similarly, $\bar{v}$ is a strict upper solution of the problem (3.8).

By monotone iteration and comparison, we obtain

$$
\bar{U}^{\star} \ll \bar{u}, \quad \bar{V}^{\star} \ll \bar{v} .
$$

For $s>0$, let $u^{s}(x)=u(x, s), v^{s}(x)=v(x, s)$ for $x \in \bar{\Omega}$. We obtain from (3.5), (3.7), (3.11), and (3.12) for $s>0$ sufficiently large

$$
u^{s}(x) \leq \bar{u}, \quad v^{s}(x) \leq \bar{v},
$$

for $x \in \bar{\Omega}$. On the other hand for $s>0$, we find from the theory of parabolic equations and the strong maximum principle that $u^{s}, v^{s}$ are in $C^{1}(\bar{\Omega})$ and

$$
u^{s}(x) \gg 0, \quad v^{s}(x) \gg 0 .
$$

Comparing (3.13) and (3.14), respectively, with (3.2) and (3.1), we obtain the conclusion of this theorem by using the first part of the proof. 
Competing interests

The authors declare that they have no competing interests.

\section{Authors' contributions}

All the authors read and approved the final manuscript.

\section{Acknowledgements}

The authors are very grateful to the anonymous referees for their valuable comments and suggestions. This work is supported by the Natural Science Foundation of Shanghai, China (No. 13ZR1430100).

Received: 1 October 2014 Accepted: 14 April 2015 Published online: 25 June 2015

\section{References}

1. Cosner, C, Laser, C: Stable coexistence states in the Volterra-Lotka competition model with diffusion. SIAM J. Appl. Math. 44, 1112-1132 (1984)

2. Dancer, $\mathrm{E}:$ On the existence and uniqueness of positive solutions for competing species models with diffusion. Trans. Am. Math. Soc. 36, 829-859 (1991)

3. Dancer, E, Guo, Z: Uniqueness and stability for solutions of competing species equations with large interactions. Commun. Appl. Nonlinear Anal. 1, 19-45 (1994)

4. Leung, A: Nonlinear Systems of Partial Differential Equations: Applications to Life and Physical Sciences. World Scientific, Singapore (2009)

5. Leung, A: Systems of Nonlinear Partial Equations. Applications to Biology and Engineering. Kluwer Academic, New York (1989)

6. Ruan, W, Pao, C: Positive steady-state solutions of competing reaction diffusion systems. J. Differ. Equ. 117, 411-427 (1995)

7. Cantrell, R, Cosner, C: Spatial Ecology via Reaction-Diffusion Equations. Wiley, New York (2003)

8. He, X, Ni, W: The effects of diffusion and spatial variation in Lotka-Volterra competition-diffusion system I: heterogeneity vs. homogeneity. J. Differ. Equ. 254, 528-546 (2013)

9. $\mathrm{He}, \mathrm{X}, \mathrm{Ni}, \mathrm{W}$ : The effects of diffusion and spatial variation in Lotka-Volterra competition-diffusion system II: the general case. J. Differ. Equ. 254, 4088-4108 (2013)

10. Álvarez-Caudevilla, P, Du, Y, Peng, R: Qualitative analysis of a cooperative reaction-diffusion system in a spatiotemporally degenerate environment. SIAM J. Math. Anal. 46, 499-531 (2014)

11. Pao, C: Nonlinear Parabolic and Elliptic Equations. Plenum, New York (1992)

\section{Submit your manuscript to a SpringerOpen ${ }^{\circ}$ journal and benefit from:}

- Convenient online submission

Rigorous peer review

- Immediate publication on acceptance

- Open access: articles freely available online

- High visibility within the field

- Retaining the copyright to your article 